\title{
Skeletal Muscle Atrophy is Exacerbated By Steatotic and Fibrotic Liver-Derived TNF-Alpha in Senescence-Accelerated Mice
}

\section{Yohei Shirakami ( $\nabla$ ys2443@gifu-u.ac.jp )}

Gifu University Graduate School of Medicine

Junichi Kato

Gifu University Graduate School of Medicine

Toshihide Maeda

Gifu University Graduate School of Medicine

Takayasu Ideta

Gifu University Graduate School of Medicine

Hiroyasu Sakai

Gifu University Graduate School of Medicine

Masahito Shimizu

Gifu University Graduate School of Medicine

\section{Research Article}

Keywords: NAFLD/NASH, steatohepatitis, sarcopenia, skeletal muscle, TNF-alpha

Posted Date: January 14th, 2022

DOI: https://doi.org/10.21203/rs.3.rs-1213317/v1

License: () (1) This work is licensed under a Creative Commons Attribution 4.0 International License.

Read Full License 


\section{Abstract}

Although liver diseases, including non-alcoholic steatohepatitis (NASH), are associated with skeletal muscle atrophy, the mechanism behind their association has not been fully elucidated. In this study, the effects of aging and NASH on the skeletal muscle and the interaction between the liver and muscle were investigated using a diet-induced NASH model in senescence-accelerated mice (SAM). A total of four groups of SAM and its control mice were fed either an NASH-inducing or control diet. In the SAM/NASH group, the histopathology of NASH and markers of oxidative stress were significant. Skeletal muscles were also markedly atrophied. The expression of the ubiquitin ligase Murf 1 in the muscle was significantly increased with muscle atrophy, while that of Tnfa was not significantly different. In contrast, the hepatic Tnfa expression and serum TNF-a levels were significantly increased in the SAM/NASH group. These results suggest that liver-derived TNF-a might promote muscle atrophy associated with steatohepatitis and aging through Murf-1. The metabolomic analysis of skeletal muscle indicated higher spermidine and lower tryptophan levels in the NASH-diet group. The findings of this study revealed an aspect of liver-muscle interaction, which might be important in developing treatments for sarcopenia associated with liver diseases.

\section{Introduction}

In recent years, the loss of skeletal muscle mass, known as sarcopenia, has attracted significant attention. Although sarcopenia was initially defined as skeletal muscle atrophy or depletion which occurs with aging ${ }^{1}$, studies have revealed that it is associated with a poor prognosis for various diseases, including hepatic diseases ${ }^{234}$. In particular, patients with liver cirrhosis have a poor prognosis because sarcopenia and obesity may develop as co-morbidities ${ }^{5}$. In addition, non-alcoholic steatohepatitis (NASH) has been reported to correlate with the progression of sarcopenia ${ }^{6}$.

The pathogenesis of sarcopenia is considered multifactorial and is caused by an imbalance between the synthesis and degradation of muscle proteins. Factors regulating muscle mass include exercise, cytokines, myostatin, cellular energy status, and factors concerning the endocrine system, including insulin resistance and the levels of circulating insulin-like growth factor (IGF), corticosteroids, and testosterone ${ }^{47}$. Because patients with chronic liver disease have abnormalities in their nutritional, metabolic, and biochemical status, liver diseases are recognised as common causes of secondary sarcopenia. Although sarcopenia is one of the major complications of chronic liver disease and the interaction between the liver and skeletal muscle has been studied, their association has not been fully understood.

The present study was conducted to elucidate the mechanism underlying NASH-related muscle atrophy using a diet-induced NASH mouse model. In addition, a senescence-accelerated mouse (SAM) ${ }^{8}$ model was employed to compare the alterations of disease state in the liver and skeletal muscle between aged and steatohepatitic mice, and to investigate the additive effects of aging on the liver and muscle. Because liver diseases alter the metabolic status of the whole body, metabolome analysis of the skeletal 
muscle was performed in the mouse model to examine changes in the metabolites under the condition of muscle atrophy.

\section{Materials And Methods}

Animals and Diets. Male senescence-accelerated mouse prone (SAMP) and senescence-accelerated mouse resistant (SAMR) were purchased from Japan SLC, Inc. (Shizuoka, Japan) and maintained at the Gifu University Animal Facility under controlled conditions of humidity $(50 \pm 10 \%)$, light (12/12 h light/dark cycle), and temperature $\left(23 \pm 2^{\circ} \mathrm{C}\right)$ according to the institutional animal care guidelines. All mice were housed in plastic cages with free access to drinking water (tap water) and maintained with a cholinedeficient, L-amino acid-defined, high-fat diet (CDAHFD, \#A06071302, Research Diets, Inc., New Brunswick, NJ, USA) or a control diet (\#A06071314, Research Diets, Inc.). CDAHFD has been reported to be an NASHinducing diet in a previous paper ${ }^{9}$. All methods are reported in accordance with ARRIVE guidelines (https://arriveguidelines.org) and were carried out in accordance with relevant guidelines and regulations.

Experimental Procedure. Fifteen SAMR and 15 SAMP were quarantined for the first seven days, following which they were separated into several groups. Six SAMRs in Group 1 and six SAMPs in Group 2 were fed the control diet. Nine SAMRs and nine SAMPs were in Groups 3 and 4, respectively, and they were all given CDAHFD. All mice at 20 weeks of age were euthanised after $12 \mathrm{~h}$ of fasting. Blood samples were collected from the inferior vena cava for clinical chemistry, and organs and tissues, including the liver, tibialis anterior, and gastrocnemius muscles, were removed for histopathological and biological examinations. The experimental protocol was approved by the Committee of Institutional Animal Experiments of Gifu University (authorization code 2020-267).

Blood Biochemistry. The whole blood was centrifuged, and serum was obtained and used for chemical analyses. Serum alanine aminotransferase (ALT) levels were determined at a commercial laboratory (SRL, Inc., Tokyo, Japan). As a marker for oxidative stress in the liver, the levels of 8-hydroxy-2'-deoxyguanosine (8-OHdG) were determined using an ELISA kit (NIKKEN SEIL Co. Ltd., Shizuoka, Japan). Serum IGF-1 and TNF-a levels were measured using the IGF-1 Mouse ELISA Kit (Thermo Fisher Scientific Inc., Waltham, MA, USA) and Mouse TNF-alpha Quantikine ELISA Kit MTA00B (R\&D Systems, Inc., Minneapolis, MN, USA), respectively, in accordance with the manufacturer's protocols.

Histological Analysis and Immunohistochemistry. For histological evaluation, the liver and muscle tissues were fixed in $10 \%$ buffered formalin, embedded in paraffin, and stained with haematoxylin and eosin. Sirius red staining was performed to determine the presence of fibrosis in the liver. Histological features of the liver were evaluated using the non-alcoholic fatty liver disease (NAFLD) activity score (NAS) system ${ }^{1011}$.

RNA extraction and quantitative real-time reverse transcription-PCR analysis. Total RNA was isolated from the liver and skeletal muscle of the experimental mice using the Rneasy Mini Kit (QIAGEN, Venlo, Netherlands). cDNA was synthesised from total RNA using a High Capacity cDNA Reverse Transcription 
Kit (Applied Biosystems, Foster City, CA, USA). Quantitative real-time reverse transcription-PCR (RT-PCR) analysis was performed using the LightCycler 96 System (Roche Diagnostics, Indianapolis, IN, USA) with LightCycler 480 SYBR Green I Master Mix (Roche Diagnostics). The specific primers used for amplifying Asma, Atrogin 1, Col1a1, F4/80, Igf1, Inos, Murf1, Tgfb1, Tnfa, and glyceraldehyde-3-phosphate dehydrogenase (Gapdh) genes were obtained from previous reports 12131415 designed using PrimerBLAST (https://www.ncbi.nlm.nih.gov/tools/primer-blast/). The sequences of the primers are listed in Table 1. The expression levels of these genes were normalised to the level of Gapdh.

Table 1

Primer sequences.

\begin{tabular}{|lll|}
\hline Target gene & Forward & Reverse \\
\hline Asma & ctctcttccagccatcttcat & tataggtggtttcgtggatgc \\
\hline Atrogin1 & GCAAACACTGCCACATTCTCTC & CTTGAGGGGAAAGTGAGACG \\
Col1a1 & catgttcagctttgtggacct & gcagctgacttcagggatgt \\
\hline F4/80 & ACAAGACTGACAACCAGACGG & TAGCATCCAGAAGAAGCAGGCGA \\
Gapdh & GACATCAAGAAGGTGGTGAAGCAG & ATACCAGGAAATGAGCTTGACAAA \\
Igf1 & tcggcctcatagtacccact & acgacatgatgtgtatctttattgc \\
\hline Inos & CGAAACGCTTCACTTCCAA & TGAGCCTATATTGCTGTGGCT \\
\hline Murf1 & ACCTGCTGGTGGAAACATC & CTTCGTGTTCCTTGCACATC \\
\hline Tgfb1 & ACCGGAGAGCCCTGGATACCA & TATAGGGGCAGGGTCCCAGACA \\
\hline Tnfa & TGGCCCAGACCCTCACACTCAG & ACCCATCGGCTGGCACCACT \\
\hline
\end{tabular}

Metabolome Analysis. The metabolome measurements were carried out at Human Metabolome Technologies, Inc. (Tsuruoka, Japan), and the concentrations of the targeted metabolites were measured and analysed as previously described ${ }^{16}$. Briefly, approximately $50 \mathrm{mg}$ of frozen skeletal muscle was cast into $50 \%(\mathrm{v} / \mathrm{v})$ acetonitrile in Milli-Q water with $20 \mu \mathrm{M}$ internal standards. The tissue was homogenised and the homogenate was centrifuged. Subsequently, the upper aqueous layer was filtered by centrifugation to remove the proteins. The filtrate was concentrated and resuspended in Milli-Q water for capillary electrophoresis time-of-flight mass spectrometry (CE-TOFMS) and capillary electrophoresistriple quadrupole mass spectrometry (CE-QqQMS) analysis using an Agilent system (Agilent Technologies, Santa Clara, CA, USA). Peaks identified in CE-TOF/MS and CE-QqQMS analyses were extracted using automatic integration software (MasterHands version 2.17.1.11, Keio University) ${ }^{17}$ and MassHunter Quantitative Analysis B.06.00 service pack (Agilent Technologies), respectively.

Statistical Analyses. Hierarchical cluster analysis (HCA) and principal component analysis (PCA) were performed using the statistical software developed by Human Metabolome Technologies Inc. To compare specific groups, one-way analysis of variance (ANOVA) was used following Shapiro-Wilk normality test. If 
the ANOVA exhibited significant differences, the Tukey-Kramer multiple comparison test was performed on items to confirm statistical significance. Kruskal-Wallis test and following Steel-Dwass test were performed for non-parametric statistical analysis. Data were presented as mean \pm standard deviations and statistical significance was set at $P<0.05$.

\section{Results}

General observations. The body weight change and the liver weight of the mice in all groups at the end of the study are shown in Fig. 1. The body weights of the mice in groups 2 and 3 were significantly lower than those of the group 1 mice. The body weights of the mice in group 4 were significantly lower than those of the group 3 mice. The relative liver weights of the mice in group 3 were significantly higher than those of the group 1 mice. The relative liver weights of the mice in group 4 were significantly higher than those in groups 2 and 3 . The results indicate that the weights of the body and liver decreased and increased, respectively, due to aging and ingestion of CDAHFD.

Aged mice develop severe steatohepatitis due to NASH-inducing diet. The livers of the SAMP fed control diet showed slight steatosis (Fig. 2A). However, there was no significant difference in the score between non-aged and aged mice that were provided with the control diet (Fig. 2B). In contrast, the livers of aged mice that were provided with the NASH-inducing diet exhibited massive macrovesicular steatosis, and the NAS was markedly higher than that of control mice fed with the same diet (Fig. 2B).

\section{Senescence exacerbates hepatic fibrosis following ingestion of NASH-inducing diet. Hepatic fibrosis} caused by the NASH-inducing diet was also enhanced in the aged mice (Fig. 2A). With regard to the hepatic fibrosis score according to a previous report ${ }^{10}$, the scores of mice fed with the NASH-inducing diet CDAHFD were significantly higher than those that were provided with the control diet (Fig. 2C). In addition, the score was markedly higher in aged mice than in control mice, both of which were fed CDAHFD, suggesting that senescence exacerbates hepatic fibrosis. The expression of genes related to fibrosis, including Asma, Col1a1, and Tgfb, was elevated in mice fed with CDAHFD compared to those given a control diet, and several genes were also significantly upregulated in aged mice (Fig. 2D).

Senescence enhances diet-induced oxidative stress and inflammation in the liver. The mRNA expression levels of Inos, which represents the degree of oxidative stress, were markedly higher due to CDAHFD in aged mice than in control mice. With regard to inflammatory cytokines, the expression of $F 4 / 80$ and $T n f a$ was also significantly upregulated following CDAHFD in aged mice compared to that in the control (Fig. $3 \mathrm{~A})$. The levels of 8-OHdG, a marker of oxidative stress, were significantly higher in the livers of control mice fed with CDAHFD compared to those fed the control diet, and the levels in aged mice fed with CDAHFD were markedly higher than those in aged and non-aged mice fed with the control diet and CDAHFD, respectively (Fig. 3B). Similar to the difference in 8-OHdG levels, the serum ALT levels of the mice were markedly higher because both non-aged and aged mice were fed with an NASH-inducing diet (Fig. 3C). Although there was no significant difference in the ALT levels between non-aged and aged mice fed with the control diet, the levels in aged mice administered with CDAHFD were significantly elevated 
compared to those in non-aged mice. These results suggest that ingesting an NASH-inducing diet causes oxidative stress, hepatic damage, and inflammation, which is enhanced in aged mice.

Serum levels of IGF-1 and TNF- $a$ in the experimental mice. The IGF-1 and TNF-a signaling pathways are involved in protein synthesis and skeletal muscle atrophy, respectively. Therefore, we examined the levels of circulating IGF-1 and TNF-a in the experimental mice. Although there was no significant difference in the serum IGF-1 levels among all groups, the levels of serum TNF-a were markedly elevated in mice fed with CDAHFD compared to those fed with the control diet. In the mice fed with CDAHFD, serum TNF-a concentrations in aged mice were significantly higher than those in non-aged mice (Fig. 3D). The results indicated that while aging and consuming an NASH-inducing diet had no effect on serum IGF-1, they led to elevated TNF-a levels.

Senescence and NASH-inducing diet causes skeletal muscle atrophy in mice. We investigated the effects of aging and an NASH-inducing diet on the skeletal muscles of mice. Comparing between the non-aged mice that were fed the control diet and CDAHFD, and between aged mice that were fed the control diet and CDAHFD, the relative weights of skeletal muscles, tibialis anterior, and gastrocnemius of mice fed with the NASH-inducing diet were significantly lower than those of mice fed with the control diet (Fig. 4A). In addition, the muscle weights of aged mice that were administered with CDAHFD were markedly decreased compared to those in non-aged mice administered with CDAHFD (Fig. 4A). Examining the muscle fibre areas in the tibialis anterior, smaller muscle fibre areas were observed in both non-aged and aged mice fed with CDAHFD (Figs. 4B and 4C). In addition, the muscle fibre areas of aged mice fed with CDAHFD were significantly smaller than in the non-aged mice fed with CDAHFD (Figs. 4B and 4C).

\section{Expression levels of ubiquitin ligases, Igf1, and Tnfa in skeletal muscles of experimental mice. The} ubiquitin ligases atrogin-1 and muscle RING-finger protein (MuRF)-1 are important regulators of ubiquitinrelated protein degradation in skeletal muscles, thereby contributing to muscle atrophy ${ }^{18}$. In this study, the mRNA expression levels of ubiquitin ligases, Atrogin 1 and Murf1, growth factor Igf1, and proinflammatory cytokine Tnfa in skeletal muscles were determined by qRT-PCR. There was no significant difference in the levels of Atrogin1 among all groups (Fig. 4D). On the other hand, the levels of Murf1 were markedly increased in skeletal muscles of mice fed CDAHFD compared to their control groups. In addition, in mice administered with CDAHFD, the levels of Murf1 in aged mice were significantly higher than those in non-aged mice. There was no significant difference in the levels of Igf1 and Tnfa among the groups.

Aging and NASH-inducing diet causes metabolic alterations in skeletal muscles. In previous studies, metabolome analyses using skeletal muscles have been performed to investigate aging-related metabolic alterations in muscles ${ }^{19}$. In this study, we also performed the metabolome analysis of mouse skeletal muscles to examine the effects of aging and an NASH-inducing diet on the muscles, and to investigate whether the metabolic alterations were related to skeletal muscle atrophy. Figs. 5A and 5B show a principal component analysis (PCA) of metabolomics and a heat map of targeted metabolites in skeletal muscles from all the experimental groups of mice. According to the PCA and heat map, it was suggested 
that the metabolites in skeletal muscles were altered by aging and consuming an NASH-inducing diet, and that there appeared to be similar alterations in aged mice fed with the control diet and non-aged mice fed with the NASH-inducing diet. Subsequently, a more detailed analysis of individual metabolites revealed that several metabolites, including spermidine and tryptophan, were affected by aging or consuming CDAHFD (Fig. 5C). Among them, the levels of spermidine and tryptophan were significantly up- and down-regulated, respectively, due to the NASH-inducing diet in non-aged mice. The levels of spermidine also tended to be higher in aged mice than in non-aged mice fed with a control diet.

\section{Discussion}

Sarcopenia is known as one of the major complications of liver diseases. Recent studies have revealed that aging contributes to an increased prevalence of NAFLD/NASH and that sarcopenia is associated with the progression of NASH; however, the detailed mechanisms through which hepatic diseases lead to skeletal muscle atrophy are not fully understood. In this study, the impact of aging and steatohepatitis on skeletal muscle atrophy was investigated using an SAM model fed with an $\mathrm{NASH}$-inducing diet. The findings of the present study demonstrated the potential mechanism through which TNF-a, produced in the liver showing steatosis and fibrosis, might induce skeletal muscle atrophy by enhancing the ubiquitinrelated pathway.

The most noteworthy aspect of this study was the inter-organ crosstalk between the liver and muscle through an inflammatory cytokine. Another interesting point might be the influence of senescence on steatohepatitis. Because aging is reported as an independent risk factor for NASH progression, researchers have attempted to reveal the interaction between aging and NAFLD/NASH. Senescent cells in the liver have recently attracted attention because cell senescence plays a role in age-associated dysfunction in various organs, including the liver. Using p16 as a marker for senescent cells, these cells were isolated, and specific removal or elimination of senescent cells in the NAFLD/NASH mouse models resulted in improved hepatic disease states ${ }^{2021}$. In a study comparing young and elderly mice, Ishibashi et al. reported that aging exacerbates steatohepatitis due to alterations in hepatic lipid metabolism; elderly mice fed with a high-fat diet exhibited severe steatohepatitis and lipotoxicity, increased inflammation, and enhanced oxidative stress in the liver ${ }^{22}$. Consistent with the results of the aforementioned study, this study indicated that aged mice fed with an NASH-inducing diet showed exacerbated steatohepatitis and fibrosis and enhanced oxidative stress in the liver compared to non-aged mice. These results suggest that aged mice are susceptible to the development of diet-induced steatohepatitis.

Metabolome analysis has contributed to novel discoveries in diseases or abnormal states of various organs, including skeletal muscle ${ }^{16192324}$. In this study, we examined the changes in metabolites in the skeletal muscle by comprehensive analysis using CE-TOFMS and CE-QqQMS in order to compare between aged and non-aged mice and between mice fed with an NASH-inducing diet and its control diet. Interestingly, PCA and hierarchical cluster analysis showed metabolic alterations in skeletal muscles due 
to aging and consuming an NASH-inducing diet, and similarity of metabolic changes in aged mice fed with the control diet and non-aged mice fed with the NASH-inducing diet.

Subsequent analyses for individual metabolites, however, revealed that only a few metabolites showed significant differences among the experimental groups, which appeared to be due to the small number of samples in each group. Among the metabolites exhibiting differences, spermidine and tryptophan were detected with a statistically significant difference. Although it has been reported that spermidine, a type of polyamine, is decreased in atrophic skeletal muscle in aged mice and supplementation with polyamine may improve sarcopenia ${ }^{19}$, spermidine in this study was increased in atrophic muscles. This discrepancy was probably caused by the differences in study design and animal model. We could not provide a conclusive answer to why spermidine was increased, but we suspect that polyamine utilization, which is required for muscle growth and maintenance, was impaired. Because details of the relationship between intramuscular polyamine levels and muscle atrophy are not fully understood ${ }^{25}$, further research is required to investigate the exact role of polyamine in skeletal muscles. Tryptophan is an important amino acid that maintains the skeletal muscle ${ }^{16}$. In this study, the levels of muscular tryptophan was found to be decreased in atrophic muscle, and a marked difference was observed between non-aged mice that were fed with the NASH-inducing and control diets. There was no significant difference in tryptophan levels among aged mice, regardless of diet. These findings suggested that tryptophan is associated with skeletal muscle atrophy induced by CDAHFD in non-aged mice, and that age mice may already be deficient in intramuscular tryptophan and hence, CDAHFD administration did not affect the results.

In the present study, skeletal muscle atrophy, which is correlated with the degree of steatohepatitis, was observed, especially in the experimental group of aged mice that were administered an NASH-inducing diet. Protein synthesis and degradation in the skeletal muscle are regulated mainly by IGF-1 and TNF-a signalling, where IGF-1 functions for synthesis through PI3K/mTOR and TNF-a induces degradation by enhancing ubiquitin ligase ${ }^{1826}$. Among the ubiquitin ligases, MuRF-1 is known to be regulated by TNF-a, while atrogin- 1 is controlled for forkhead box-containing protein (FoxO) which is negatively regulated by IGF- ${ }^{18}$. In this study, there were no significant differences in the mRNA expression levels of Igf1 in the liver and muscle, and the serum IGF-1 concentration among all groups, suggesting that skeletal muscle atrophy in this mouse model was not related to IGF-1 signalling. However, the levels of hepatic Tnfa mRNA expression and serum TNF-a were increased in aged and CDAHFD-fed mice, although the Tnfa expression in muscle showed no significant differences among groups. These findings suggested that skeletal muscle atrophy is induced by TNF- $a$, which is transported from the liver with steatosis and inflammation.

The role of TNF-a in skeletal muscle atrophy has been extensively investigated ${ }^{272829303132}$. In one study, the authors demonstrated that TNF-a produced in fibrotic liver was transported to muscle through the bloodstream, leading to atrophy of skeletal muscle. They also conducted an in vitro study using a muscle cell line and serum from mice with liver fibrosis, indicating an exclusive impact of serum TNF- $a$ on myotube atrophy. Consistent with the findings of the aforementioned study, the results from this study 
revealed that TNF- $a$ acts as an important cytokine to induce hepatic disease-related skeletal muscle atrophy.

In summary, the present study indicated that senescent liver is susceptible to steatosis and fibrosis due to enhanced oxidative stress, and that TNF-a released from steatotic and fibrotic liver might induce NAFLD/NASH-associated skeletal muscle atrophy (Fig. 6). As was also suggested by a previous report ${ }^{30}$, this liver-muscle crosstalk has clinical significance because TNF-a is considered as one of the essential factors for skeletal muscle atrophy promoted by hepatic diseases, and this circulating cytokine can be a potential target for the prevention and treatment of NAFLD/NASH-associated skeletal muscle atrophy.

\section{Abbreviations}

ALT, alanine aminotransferase; ANOVA, analysis of variance; CDAHFD, choline-deficient, L-amino aciddefined, high-fat diet; CE-TOFMS, capillary electrophoresis time-of-flight mass spectrometry; CE-QqQMS, capillary electrophoresis-triple quadrupole mass spectrometry; FoxO, forkhead box-containing protein; HCA, hierarchical cluster analysis; HE, hematoxylin and eosin; IGF, insulin-like growth factor; NAFLD, nonalcoholic fatty liver disease; NAS, non-alcoholic fatty liver disease activity score; NASH, non-alcoholic steatohepatitis; PCA, principal component analysis; qRT-PCR, quantitative transcription PCR; SAM, senescence-accelerated mouse; SAMP, senescence-accelerated mouse prone; SAMR, senescenceaccelerated mouse resistant; TNF, tumour necrosis factor; 8-OHdG, 8-hydroxy-2'-deoxyguanosine

\section{Declarations}

\section{Acknowledgments}

We are grateful to Chiyoko Sano, Akihiro Abe, Toshiki Ohta, Aya Nagai, and Chihiro Shiba for their technical assistance. The authors also would like to thank the Society for Senescence-Accelerated Mouse (SAM) Research for sharing the mice and Editage (www.editage.com) for English language editing.

\section{Author Contributions}

Y.S. J.K., and M.S. conceived and designed the experiments. Y.S., J.K., T.M., T.I., and H.S. performed the experiments. Y.S., J.K., and H.S. analyzed the data. Y.S. and J.K. wrote the main manuscript text. Y.S., J.K. T.M., and T.I. were involved in preparing all figures and tables. All authors reviewed the manuscript.

\section{Funding}

This work was supported by JSPS KAKENHI Grant Numbers 16K09352, 19K08368, and 19K08465.

\section{Competing interests}

The authors declare no competing interests. 


\section{References}

1. Rosenberg, I. H. Sarcopenia: origins and clinical relevance. J Nutr 127, 990S-991S (1997).

2. Shachar, S. S., Williams, G. R., Muss, H. B. \& Nishijima, T. F. Prognostic value of sarcopenia in adults with solid tumours: A meta-analysis and systematic review. Eur J Cancer 57, 58-67 (2016).

3. Ooi, P. H. et al. Sarcopenia in Chronic Liver Disease: Impact on Outcomes. Liver Transplant Off Publ Am Assoc Study Liver Dis Int Liver Transplant Soc 25, 1422-1438 (2019).

4. Ebadi, M., Bhanji, R. A., Mazurak, V. C. \& Montano-Loza, A. J. Sarcopenia in cirrhosis: from pathogenesis to interventions. J Gastroenterol 54, 845-859 (2019).

5. Hara, N. et al. Sarcopenia and Sarcopenic Obesity Are Prognostic Factors for Overall Survival in Patients with Cirrhosis. Intern Med 55, 863-870 (2016).

6. Bhanji, R. A., Narayanan, P., Allen, A. M., Malhi, H. \& Watt, K. D. Sarcopenia in hiding: The risk and consequence of underestimating muscle dysfunction in nonalcoholic steatohepatitis. Hepatology 66, 2055-2065 (2017).

7. Meyer, F. et al. Molecular Mechanism Contributing to Malnutrition and Sarcopenia in Patients with Liver Cirrhosis. Int J Mol Sci 21, (2020).

8. Hosokawa, M. A higher oxidative status accelerates senescence and aggravates age-dependent disorders in SAMP strains of mice. Mech Ageing Dev 123, 1553-1561 (2002).

9. Matsumoto, M. et al. An improved mouse model that rapidly develops fibrosis in non-alcoholic steatohepatitis. Int J Exp Patho/ 94, 93-103 (2013).

10. Kleiner, D. E. et al. Design and validation of a histological scoring system for nonalcoholic fatty liver disease. Hepatology 41, 1313-1321 (2005).

11. Lucas, C., Lucas, G., Lucas, N., Krzowska-Firych, J. \& Tomasiewicz, K. A systematic review of the present and future of non-alcoholic fatty liver disease. Clin Exp Hepatol 4, 165-174 (2018).

12. Robert, F. et al. Targeting protein synthesis in a Myc/mTOR-driven model of anorexia-cachexia syndrome delays its onset and prolongs survival. Cancer Res 72, 747-756 (2012).

13. Miyazaki, T. et al. Novel FXR agonist nelumal A suppresses colitis and inflammation-related colorectal carcinogenesis. Sci Rep 11, 492 (2021).

14. Nagano, J. et al. Effects of indoleamine 2,3-dioxygenase deficiency on high-fat diet-induced hepatic inflammation. PLoS One 8, e73404 (2013).

15. Shirakami, Y. \& Shimizu, M. Possible mechanisms of green tea and its constituents against cancer. Molecules 23, (2018).

16. Ninomiya, S. et al. Low Levels of Serum Tryptophan Underlie Skeletal Muscle Atrophy. Nutrients 12, 978 (2020).

17. Sugimoto, M., Wong, D. T., Hirayama, A., Soga, T. \& Tomita, M. Capillary electrophoresis mass spectrometry-based saliva metabolomics identified oral, breast and pancreatic cancer-specific profiles. Metabolomics 6, 78-95 (2010). 
18. Gumucio, J. P. \& Mendias, C. L. Atrogin-1, MuRF-1, and sarcopenia. Endocrine 43, 12-21 (2013).

19. Uchitomi, R. et al. Metabolomic Analysis of Skeletal Muscle in Aged Mice. Sci Rep 9, 10425 (2019).

20. Omori, S. et al. Generation of a p16 Reporter Mouse and Its Use to Characterize and Target p16(high) Cells In Vivo. Cell Metab 32, 814-828.e6 (2020).

21. Ogrodnik, M. et al. Cellular senescence drives age-dependent hepatic steatosis. Nat Commun $\mathbf{8}$, 15691 (2017).

22. Ishizuka, K. et al. Aging exacerbates high-fat diet-induced steatohepatitis through alteration in hepatic lipid metabolism in mice. J Gastroenterol Hepatol 35, 1437-1448 (2020).

23. Saoi, M. \& Britz-McKibbin, P. New Advances in Tissue Metabolomics: A Review. Metabolites 11, (2021).

24. Hatazawa, Y. et al. Metabolomic Analysis of the Skeletal Muscle of Mice Overexpressing PGC-1a. PLoS One 10, e0129084 (2015).

25. Lee, N. K. L. \& MacLean, H. E. Polyamines, androgens, and skeletal muscle hypertrophy. J Cell Physiol 226, 1453-1460 (2011).

26. Nakamura, S. et al. Insulin-like growth factor-l is required to maintain muscle volume in adult mice. $J$ Bone Miner Metab 37, 627-635 (2019).

27. Patel, H. J. \& Patel, B. M. TNF-a and cancer cachexia: Molecular insights and clinical implications. Life Sci 170, 56-63 (2017).

28. Thoma, A. \& Lightfoot, A. P. NF-kB and Inflammatory Cytokine Signalling: Role in Skeletal Muscle Atrophy. Adv Exp Med Biol 1088, 267-279 (2018).

29. Pan, L. et al. Inflammation and sarcopenia: A focus on circulating inflammatory cytokines. Exp Gerontol 154, 111544 (2021).

30. Kurosawa, T. et al. Liver fibrosis-induced muscle atrophy is mediated by elevated levels of circulating TNFa. Cell Death Dis 12, 11 (2021).

31. Kato, J., Shirakami, Y. \& Shimizu, M. Diabetes Mellitus and Colon Carcinogenesis: Expectation for Inhibition of Colon Carcinogenesis by Oral Hypoglycemic Drugs. Gastrointest Disord 1, 273-289 (2019).

32. Li, Y., Zhang, F., Modrak, S., Little, A. \& Zhang, H. Chronic Alcohol Consumption Enhances Skeletal Muscle Wasting in Mice Bearing Cachectic Cancers: The Role of TNFa/Myostatin Axis. Alcohol Clin Exp Res 44, 66-77 (2020).

\section{Figures}


A

\section{Body weight change}

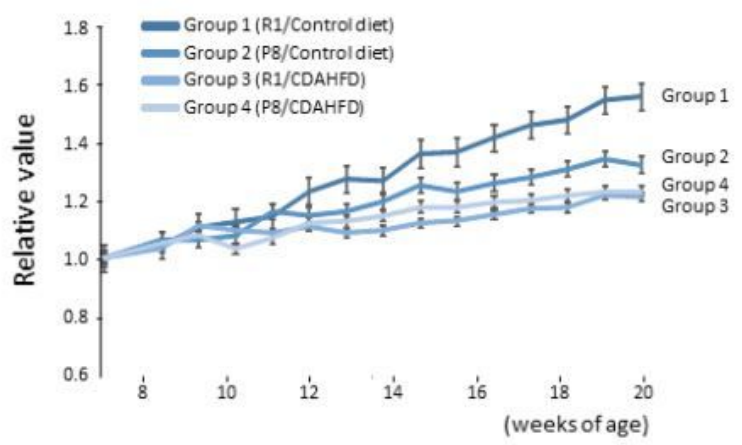

B

\section{Relative weight of liver}

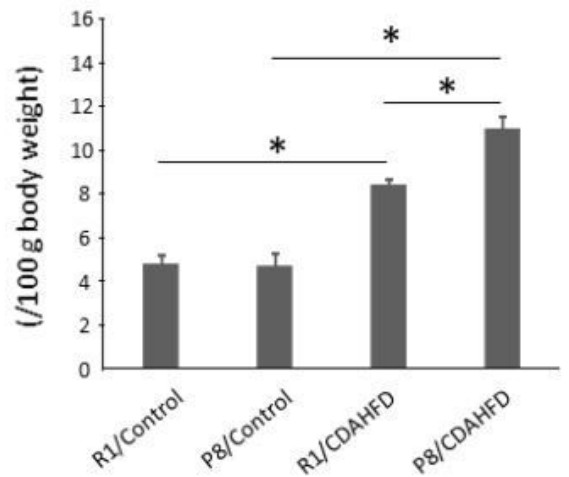

\section{Figure 1}

Body weight change and relative weights of liver. $(A)$ Body weight change of the experimental mice during the study. Weights are presented as relative to 1 at starting point. (B) Relative weights of liver of the experimental mice at the end of study. Data are the means and standard deviations. ${ }^{\star} P<0.05$. CDAHFD, cholin-deficient L-amino acid-defined high fat diet; P8, senescence-accelerated mouse prone (SAMP8, aged mouse); R1, senescence-accelerated mouse resistant (SAMR1, control mouse). 
A

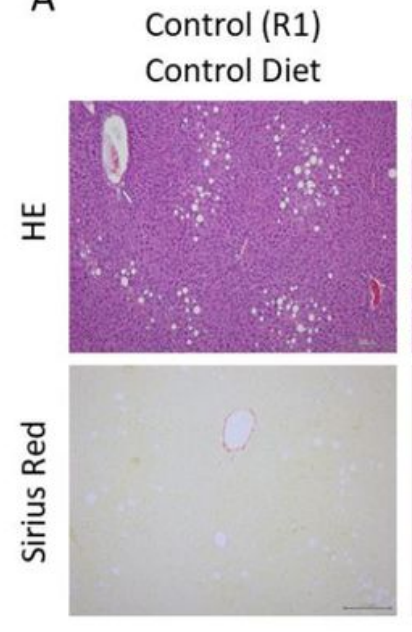

C

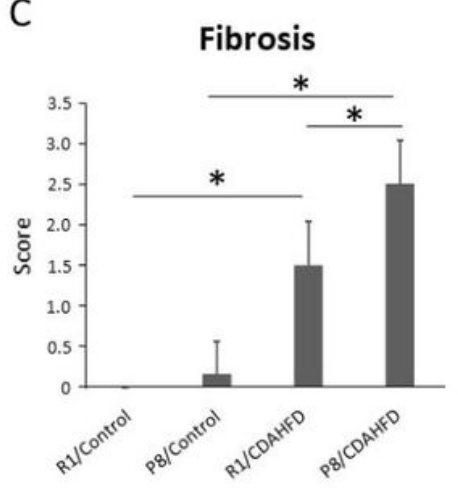

Aged (P8) Control Diet
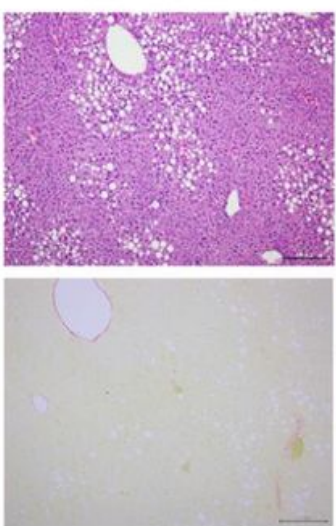

CDAHFD

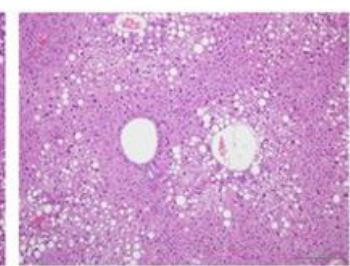

Control (R1)
Aged (P8)

CDAHFD

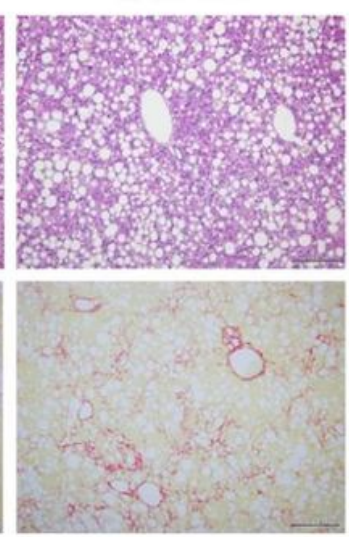

B

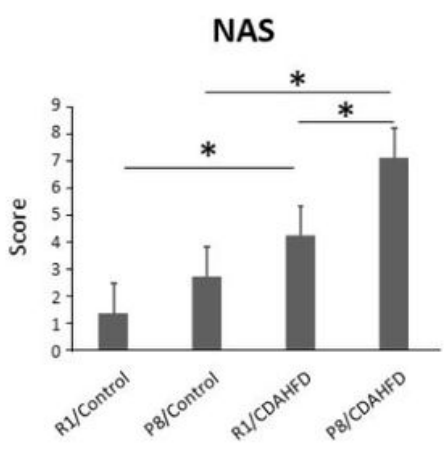

D

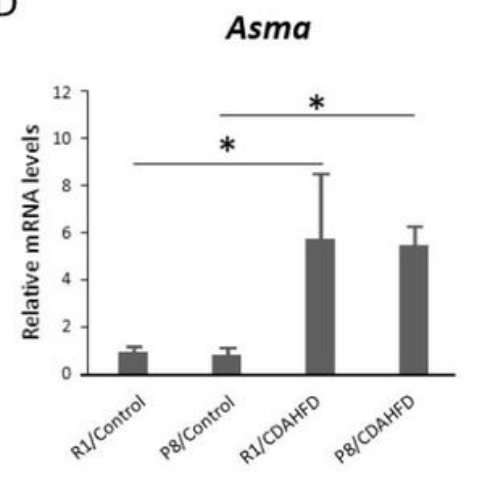

Col1a1

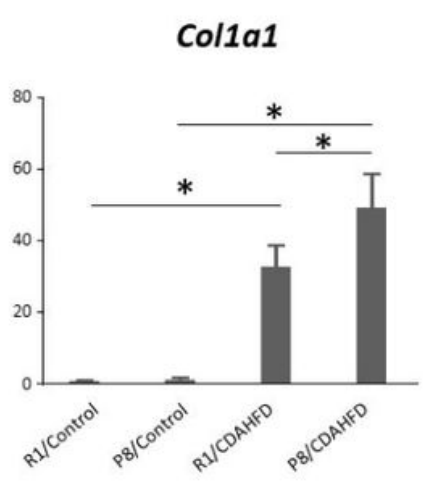

Tgfb1

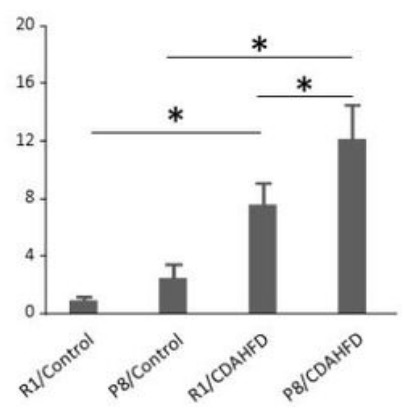

Figure 2

Effects of aging and NASH-inducing diet on hepatic steatosis and fibrosis. (A) Histopathological alterations in the liver were evaluated by staining with haematoxylin and eosin and Sirius Red. Bars, 200 $\mu \mathrm{m}$. (B) NAFLD activity scores (NAS). (C) Fibrosis scores. (D) mRNA expression levels in the liver evaluated among all groups using qRT-PCR. Data are the means and standard deviations. ${ }^{*} P<0.05$. CDAHFD, cholin-deficient L-amino acid-defined high fat diet; P8, senescence-accelerated mouse prone (SAMP8, aged mouse); R1, senescence-accelerated mouse resistant (SAMR1, control mouse). 
A
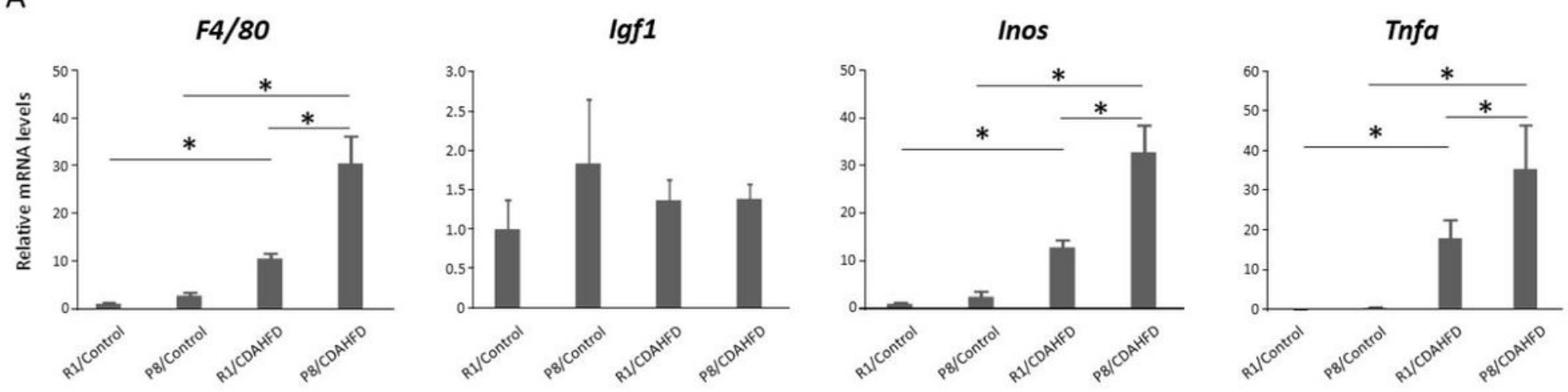

B

C

Liver 8-OHdG

Serum ALT
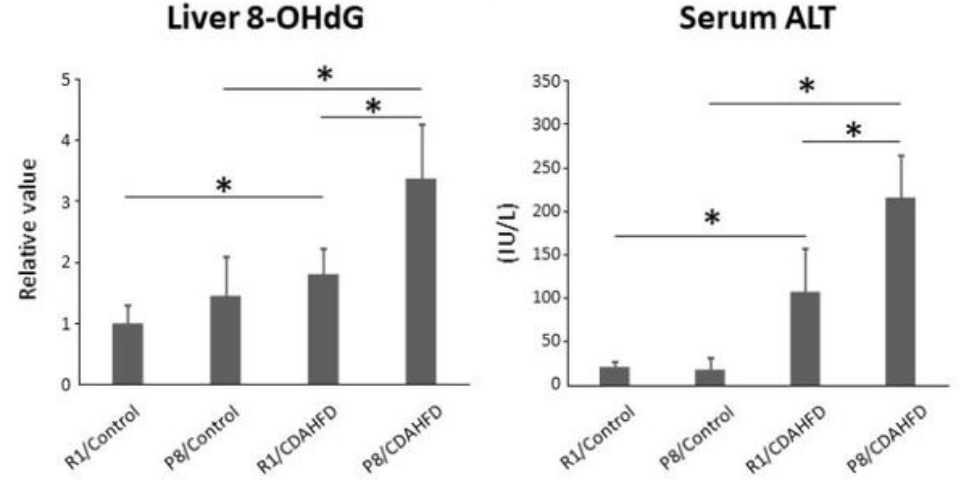

D
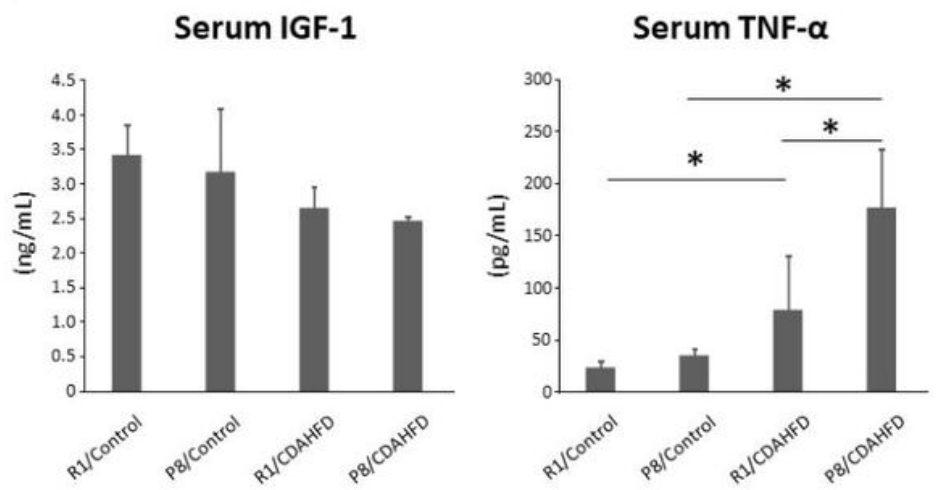

Figure 3

Effects of aging and NASH-inducing diet on inflammation and oxidative stress in liver and serum IGF-1 and TNF-a. (A) mRNA expression levels in liver were evaluated among all groups using qRT-PCR. (B) Oxidative stress in the liver evaluated using levels of 8-hydroxy-2'-deoxyguanosine (8-OHdG). (C) The values of serum alanine aminotransferase (ALT). (D) Values of serum IGF-1 and TNF-a measured using ELISA. Data are the means and standard deviations. ${ }^{\star} P<0.05$. ALT, alanine transaminase; CDAHFD, cholin-deficient L-amino acid-defined high fat diet; IGF, insulin-like growth factor, P8, senescenceaccelerated mouse prone (SAMP8, aged mouse); R1, senescence-accelerated mouse resistant (SAMR1, control mouse). 


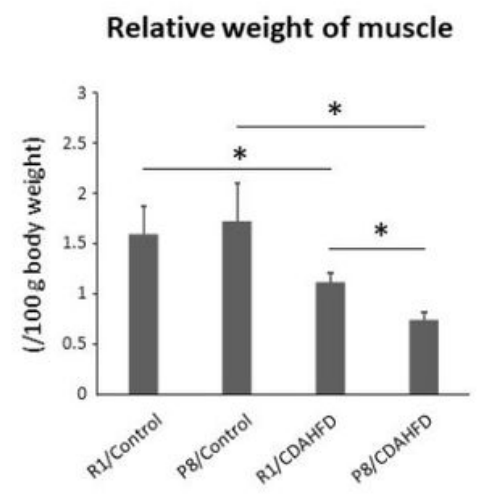

D

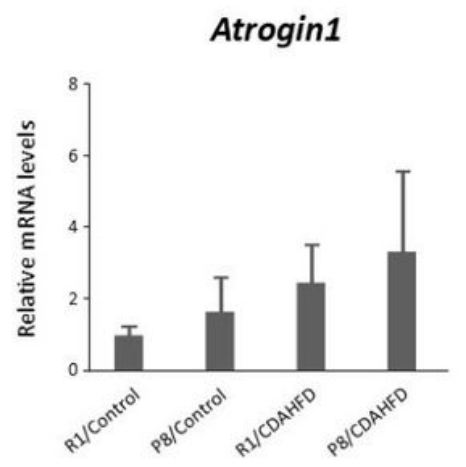

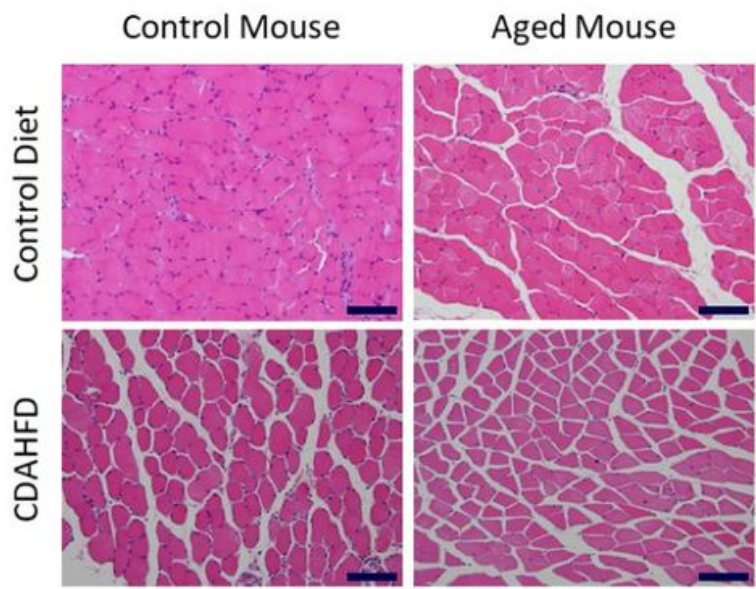

Igf1

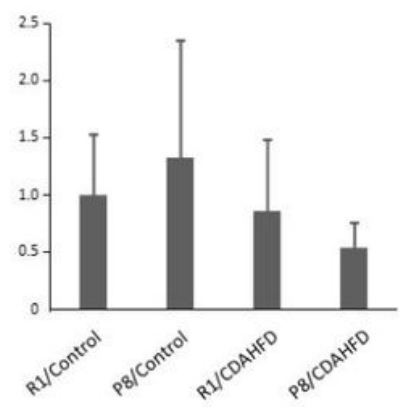

Murf1
C
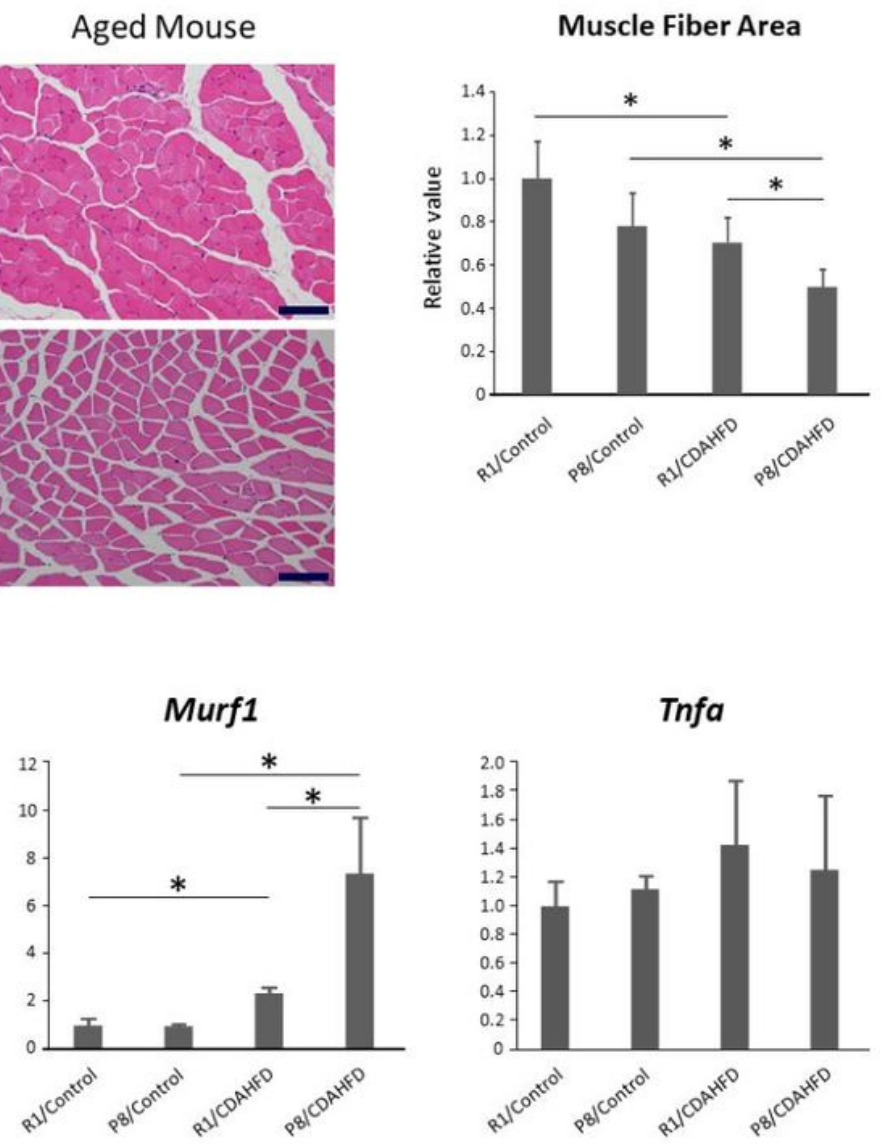

Tnfa

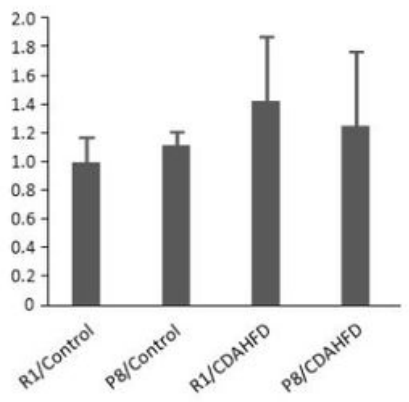

Figure 4

Effects of aging and NASH-inducing diet on skeletal muscle. (A) Relative weights of lower limb muscles, including tibialis anterior and gastrocnemius, of the experimental mice at the end of the study. (B) Histopathological alterations in tibialis anterior muscle were evaluated by staining with haematoxylin and eosin. Bars, $100 \mu \mathrm{m}$. (C) Cross-section muscle fibre area was measured. (D) mRNA expression levels in the skeletal muscle were evaluated among all groups using qRT-PCR. Data are the means and standard deviations. ${ }^{\star} P<0.05$. CDAHFD, cholin-deficient $L$-amino acid-defined high fat diet; $P 8$, senescenceaccelerated mouse prone (SAMP8, aged mouse); R1, senescence-accelerated mouse resistant (SAMR1, control mouse). 
A

Principal Component Analysis

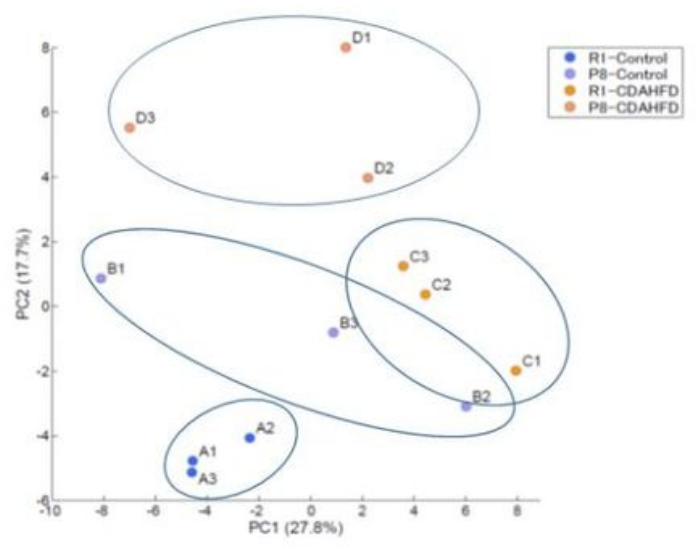

\section{B Hierarchical Cluster Analysis (heatmap)}

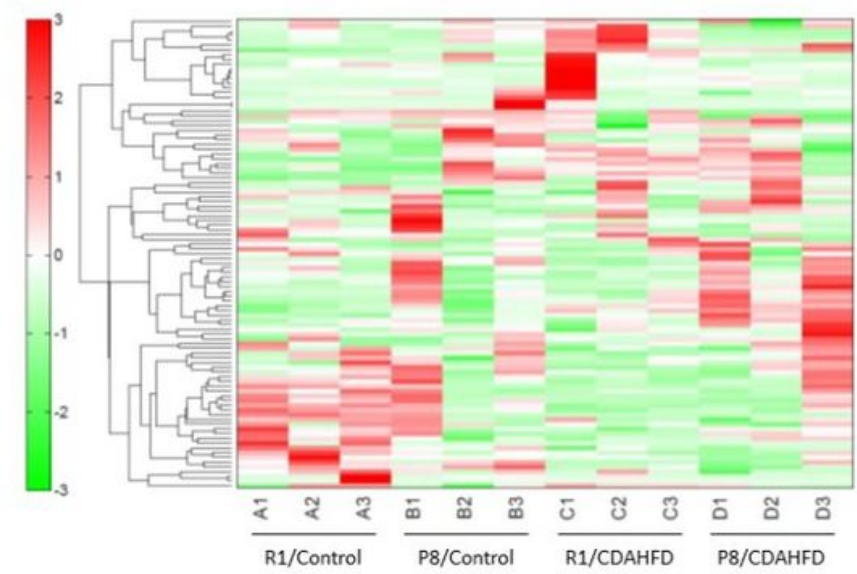

C
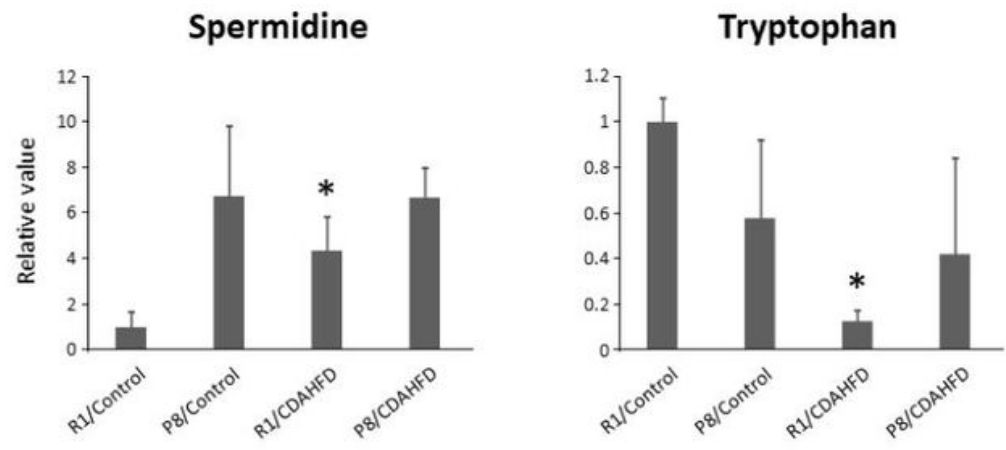

Figure 5

Metabolome analyses of skeletal muscle. (A) Principal component analysis to compare the experimental mouse groups. (B) Hierarchical cluster analysis (heatmap). (C) Comparing individual metabolite among groups. ${ }^{*} P<0.05$. CDAHFD, cholin-deficient L-amino acid-defined high fat diet; $P 8$, senescenceaccelerated mouse prone (SAMP8, aged mouse); R1, senescence-accelerated mouse resistant (SAMR1, control mouse). 


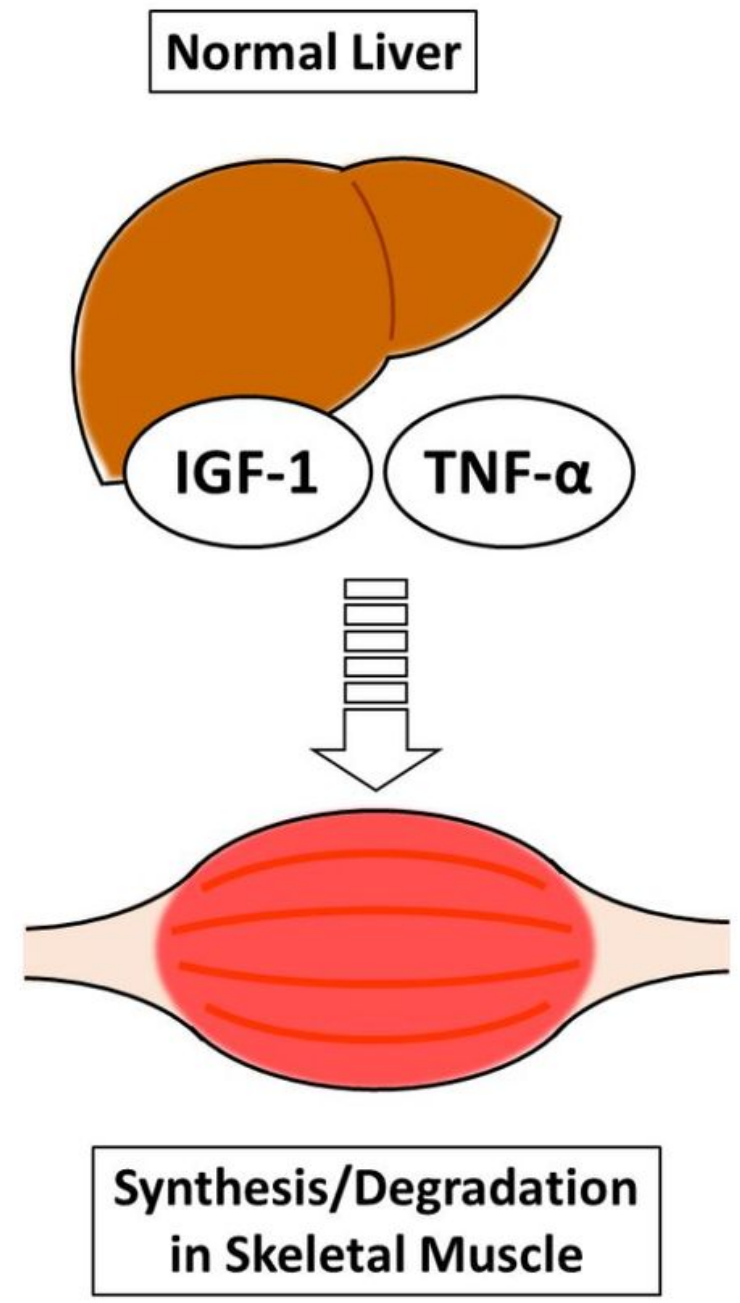

\section{Steatotic and Fibrotic Liver}
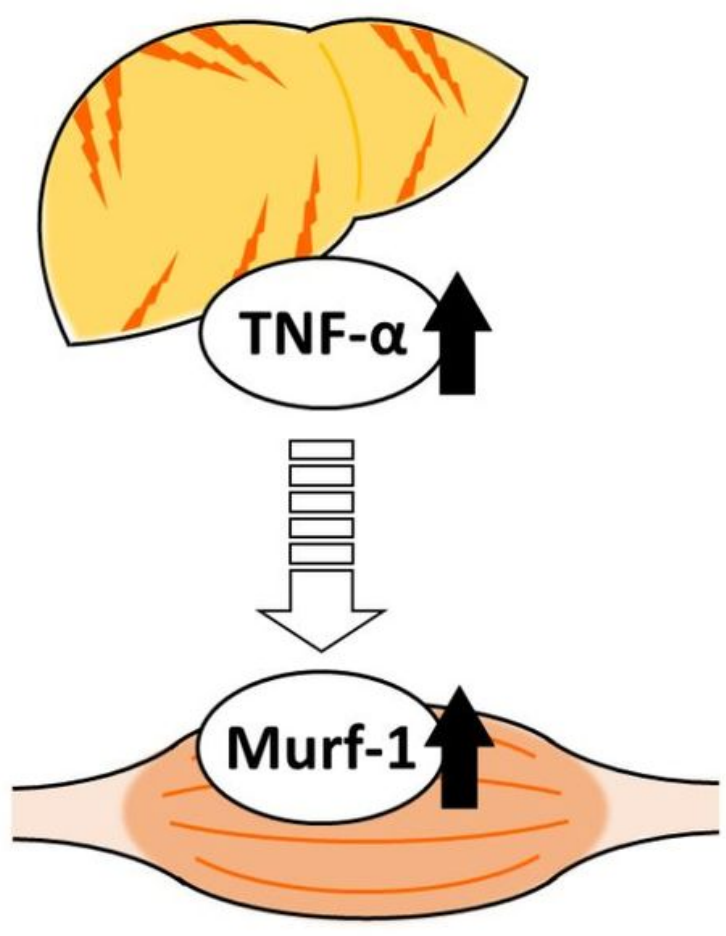

Skeletal Muscle Atrophy

Figure 6

Assumed interacting mechanism for the pathogenesis of steatotic and fibrotic liver-induced skeletal muscle atrophy. Protein synthesis and degradation in the skeletal muscle are regulated mainly by IGF-1 and TNF-a signalling, where IGF-1 functions for synthesis through PI3K/mTOR and TNF-a induces degradation by enhancing ubiquitin ligase, such as Murf-1. The results of this study indicate that skeletal muscle atrophy is exacerbated by elevated serum TNF-a, which is derived from steatotic and fibrotic liver, in mice fed an $\mathrm{NASH}$-inducing diet. 